\title{
A REVIEW ON FORMULATION AND CHARACTERIZATION OF NANOEMULSION
}

\author{
NURUL AMIN*, BISWAJIT DAS \\ Department of Pharmaceutics, Girijananda Chowdhury Institute of Pharmaceutical Science, Hatkhowapara, Azara, Guwahati-17 \\ Email: nurulamin374@gmail.com
}

Received: 14 Apr 2019, Revised and Accepted: 13 Jun 2019

\begin{abstract}
Nanoemulsions are clear, thermodynamically stable mixtures of oil, water, surfactant and co-surfactant. These are the oil-in-water type of emulsions with the average droplet size ranging from $5 \mathrm{~nm}$ to $100 \mathrm{~nm}$. Nanoemulsions are thermodynamically stable transparent or translucent dispersions of oil and water stabilized by an interfacial film of surfactant and co-surfactant molecules having a droplet size of less than $100 \mathrm{~nm}$. This review provides brief information about the method of preparation and evaluation of nanoemulsion as drug carriers for improving the delivery of therapeutic agents. There are several techniques are to be used for preparation of nanoemulsions like high pressure homogenization, microfluidization, low energy emulsification and solvent evaporation method and parameter that are to be used for its characterization like droplet size analysis, viscosity determination, drug content, refractive index, $\mathrm{pH}$, zeta potential, Transmission electron microscopy, thermal stability, release and in vitro skin permeation study.
\end{abstract}

Keywords: Nanoemulsion, Homogenization, Emulsification, Characterization, Application

(C) 2019 The Authors. Published by Innovare Academic Sciences Pvt Ltd. This is an open access article under the CC BY license (http://creativecommons.org/licenses/by/4.0/) DOI: http://dx.doi.org/10.22159/ijcpr.2019v11i4.34925

\section{INTRODUCTION [1, 2, 3]}

A nanoemulsion is considered to be a thermodynamically or kinetically stable liquid dispersion of two immiscible liquid phases such as an oil phase and a water phase. An interfacial tension exists between the two liquids everywhere they are in contact due to differences in attractive interactions between the molecules of the two liquid phases. Amphiphilic surface-active molecules or surfactants are added to reduce this interfacial tension. Droplet size of nanoemulsion falls typically in the range of $5 \mathrm{~nm}$ to $100 \mathrm{~nm}$ and shows a narrow size distribution. The key difference between emulsions and Nanoemulsions are that the former exhibit excellent kinetic stability, are fundamentally thermodynamically unstable as well as emulsions are cloudy while Nanoemulsions are clear or translucent.

Types of nanoemulsions $[4,5]$

Three types of Nanoemulsions are most likely to be formed depending on the composition:

- Oil in water Nanoemulsions wherein oil droplets are dispersed in the continuous aqueous phase;

- Water in oil Nanoemulsions wherein water droplets are dispersed in the continuous oil phase;

- Bi-continuous Nanoemulsions wherein microdomains of oil and water are interdispersed within the system.

In all three types of Nanoemulsions, the interface is stabilized by an appropriate combination of surfactants and/or co-surfactants.

The key difference between emulsions and Nanoemulsions are that the former, whilst they may exhibit excellent kinetic stability, are fundamentally thermodynamically unstable and will eventually phase separate. Another important difference concerns their appearance; emulsions are cloudy while Nanoemulsions are clear or translucent. In addition, there are distinct differences in their method of preparation, since emulsions require a large input of energy while Nanoemulsions.

\section{Advantages of nanoemulsion [6]}

Increase the rate of absorption. Eliminates variability in absorption. Helps in the solubilizing lipophilic drug. Provides aqueous dosage form for water-insoluble drugs. Increases bioavailability. Various routes like tropical, oral and intravenous can be used to deliver the product. Rapid and efficient penetration of the drug moiety. Helpful in taste masking. Liquid dosage form increases patient compliance. Less amount of energy requirement. Nanoemulsions are thermodynamically stable system and the stability allows selfemulsification of the system whose properties are not dependent on the process followed. Same Nanoemulsions can carry both lipophilic and hydrophilic drugs. The use of Nanoemulsion as delivery systems can improve the efficacy of a drug, allowing the total dose to be reduced and thus minimizing side effects.

\section{Disadvantages of nanoemulsion [6]}

Nanoemulsion stability is influenced by environmental parameters such as temperature and $\mathrm{pH}$. These parameters change upon Nanoemulsion delivery to patients. High-pressure homogenisers, ultrasonic, micro fluidiser and other special application techniques are required for nanoemulsions preparation. Such equipment (such as the Microfluidiser) became available only in recent years. Use of a large concentration of surfactant and co-surfactant necessary for stabilizing the nanodroplets. Limited solubilizing capacity for highmelting substances. The surfactant must be nontoxic for using pharmaceutical applications. High cost of commercial production especially for the cosmetic industry because of using expensive equipment as well as high concentrations of emulsifiers. It is difficult to understand the role of surfactants and cosurfactants and also the mechanism of production of submicron droplets. Classical macroemulsion systems provide better benefits than using nanoemulsions. The interfacial chemistry is important factors for the production of nanoemulsions. But the function of the interfacial chemistry is not clear. Difficult for high-melting substances due to their limited solubility.

\section{Components of nanoemulsion [7-9]}

\section{Oils}

Selection of a proper oily phase is very essential as it influences the selection of other ingredients of nanoemulsions, generally in case of $\mathrm{o} / \mathrm{w}$ nanoemulsions. Usually, the oil having greatest solubilising potential has selected as an oily phase for the formulation of nanoemulsions. This helps to attain maximum drug loading in the nanoemulsions. Naturally occurring oils and fats contains a mixture of triglycerides which contain fatty acids of varying chain lengths and degrees of unsaturation. Triglycerides are classified as short $(<5$ carbons), medium (6-12 carbons), or long-chain ( $>12$ carbons) and 
may be synthetically hydrogenated to decrease the degree of unsaturation, thereby conferring resistance to oxidative degradation. The selection of oily phase is also a compromise between its ability to solubilize the drugs and its ability to ease the formation of nanoemulsion of desired properties. Some suitable oil phases are Captex 355, Myritol 318, IPM, modified vegetable oils, digestible or non-digestible oils and fats such as olive oil, palm oil, corn oil, oleic acid, sesame oil, soybean oil, hydrogenated soybean oil, peanut oil and beeswax.

\section{Surfactants}

The surfactant should capable of micro emulsification of the oily phase and should also possess the good solubilizing potential for the hydrophobic drug compounds. The choice of the surfactant is critical for the nanoemulsion formulation. Surfactants with an HLB value $<10$ are hydrophobic (such as sorbitan monoesters) and form w/o nanoemulsion whereas high HLB $(>10)$ surfactants such as polysorbate 80 are hydrophilic and form o/w nanoemulsion. The hydrophobic core enhances the entrapment of drug, thus increasing its solubility. When the oil content is high, surfactant concentrate on the oil/water interface forming emulsions, wherein the drug is solubilized in the internal oil phase. On the other hand, when the oil content is low, minute oil entrapped surfactant globules are produced, which are known as nanoemulsions. The surfactant used in nanoemulsion formation could be ionic or non-ionic but ionic surfactants are not preferred due to toxicological effects. Various surfactants are mostly used such as lecithins, poloxamers and polysorbate 80 . It is very important to determine the surfactant concentration properly as large amounts of surfactants may cause GI irritation. There is a relationship between the droplet size and the concentration of the surfactant being used. In some cases, increasing the surfactant concentration could lead to smaller droplets such as in the case of a mixture of saturated C8-C10 polyglycolized glycerides. On the other hand, in some cases, the mean droplet size may increase with increasing surfactant concentrations.

\section{Co-surfactants}

Many times, surfactant alone cannot lower the oil-water interfacial tension adequately to yield a nanoemulsion which necessitates the addition of a co-surfactant to bring about the surface tension close to zero. Co-surfactants penetrate into the surfactant monolayer providing additional fluidity to interfacial film and thus distracting the liquid crystalline phases which are formed when surfactant film is too rigid. Usually, a very low HLB co-surfactant is used with a high HLB surfactant to modify the overall HLB of the system. Unlike surfactant, the co-surfactant may not be capable of forming selfassociated structures like micelles on its own. Hydrophilic cosurfactants preferably alcohols of intermediate chain length such as hexanol, pentanol and octanol, which are known to reduce the oil/water interface and allow the spontaneous formation of nanoemulsion.

\section{Techniques of preparation of nanoemulsions [10-12]}

Nanoemulsions have very small particle size range; they can be most effectively produced using high-pressure equipment. The most commonly used methods for producing nanoemulsions are „Highpressure homogenization and Microfluidization used at both laboratory and industrial scale. Other methods like Ultrasonification and In-situ emulsification are also suitable for preparation of nanoemulsion.

\section{High-pressure homogenization}

The preparation of nanoemulsions requires high-pressure homogenization. This technique makes use of high-pressure homogenizer/piston homogenizer to produce nanoemulsions of extremely low particle size (up to $1 \mathrm{~nm}$ ). The dispersion of two liquids (oily phase and aqueous phase) is achieved by forcing their mixture through a small inlet orifice at very high pressure (500 to $5000 \mathrm{psi})$, which subjects the product to intense turbulence and hydraulic shear resulting in extremely fine particles of emulsion. The particles which are formed exhibit a liquid, lipophilic core separated from the surrounding aqueous phase by a monomolecular layer of phospholipids. This technique has great efficiency, the only disadvantage being high energy consumption and an increase in temperature of the emulsion during processing.

\section{Advantages}

* Effectively used for thermolabile substances.

* Ease of scale-up and little batch-to-batch variation

* Narrow size distribution of the nanoparticulate drug.

* Flexibility in handling the drug quality.

\section{Microfluidization}

Microfluidization is a mixing technique, which makes use of a device called microfluidizer. This device uses a high-pressure positive displacement pump (500 to $20000 \mathrm{psi}$ ), which forces the product through the interaction chamber, which consists of small channels called „microchannels ". The product flows through the microchannels on to an impingement area resulting in very fine particles of sub-micron range. The two solutions (aqueous phase and oily phase) are combined together and processed in an inline homogenizer to yield a coarse emulsion. The coarse emulsion is into a microfluidizer where it is further processed to obtain a stable nanoemulsion. The coarse emulsion is passed through the interaction chamber microfluidizer repeatedly until the desired particle size is obtained. The bulk emulsion is then filtered through a filter under nitrogen to remove large droplets resulting in a uniform nanoemulsion.
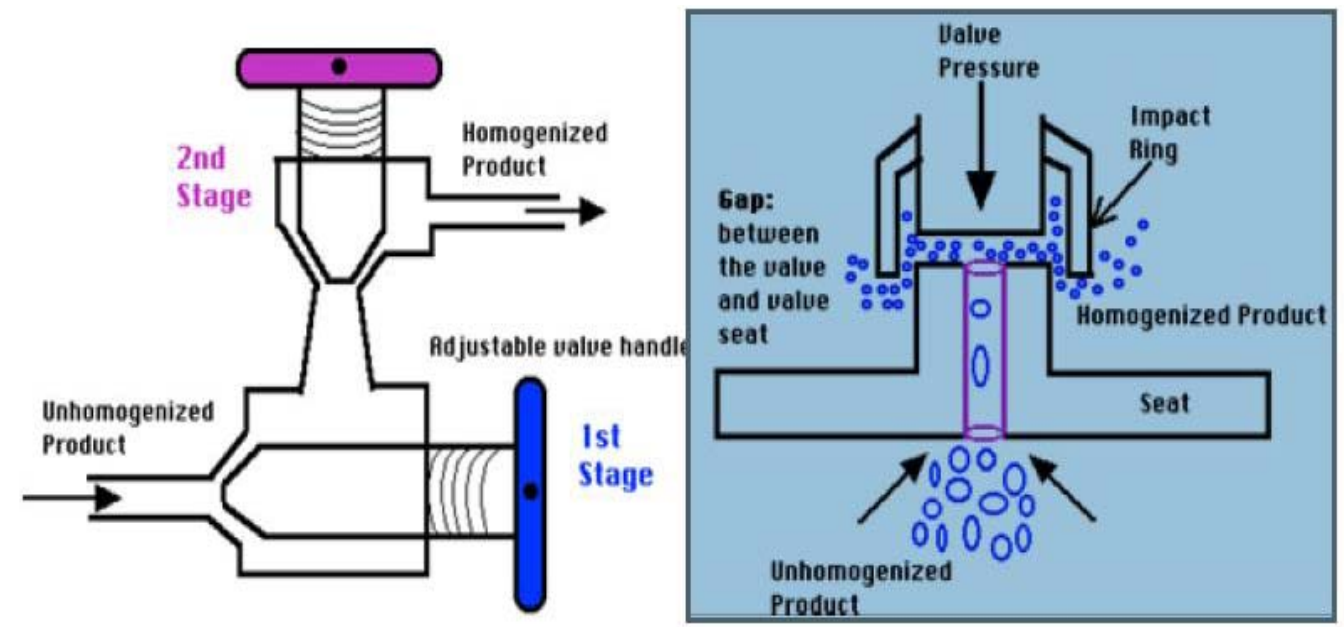

Fig. 1: Processes of microfluidization by microfluidizer 


\section{Ultrasonication}

The preparation of Nanoemulsion is reported in various research papers which aim to use the ultrasonic sound frequency for the reduction of the droplet size. Another approach is the use of a constant amplitude sonotrode at system pressures in excess of the ambient value. It is well known that increasing the external pressure increases the cavitations threshold within an ultrasonic field and thus fewer bubbles form. However, increasing the external pressure also increases the collapse pressure of cavitations bubbles. This means that the collapse of the bubbles when cavitation occurs becomes stronger and more violent than when the pressure is at atmospheric conditions. As cavitation is the most important mechanism of power dissipation in a low-frequency ultrasonic system, these changes in navigational intensity can be related directly to changes in the power density. The system also uses a water jacket to control the temperature to the optimum level.

\section{Phase inversion method}

In this method, fine dispersion is obtained by chemical energy resulting of phase transitions produced by the emulsification pathway. The phase transition is produced by varying the composition of the emulsion and keeping temperature constant or vice versa. The phase inversion temperature was first done by Shinoda et al. it was concluded that an increase in temperature results in the chemical changes of polyoxyethylene surfactants by the degradation of the polymer chain with the temperature.

Spontaneous emulsification: It involves three main steps:

- Preparation of homogeneous organic solution composed of oil and lipophilic surfactant in water-miscible solvent and hydrophilic surfactant, $>$ The organic phase was injected in the aqueous phase under magnetic stirring the $\mathrm{o} / \mathrm{w}$ emulsion was formed, $\bullet$ The water-miscible solvent was removed by evaporation under reduced pressure.

\section{Solvent evaporation technique}

This technique involves preparing a solution of drug followed by its emulsification in another liquid that is non-solvent for the drug. Evaporation of the solvent leads to precipitation of the drug. Crystal growth and particle aggregation can be controlled by creating high shear forces using a high-speed stirrer.

\section{Hydrogel method}

It is similar to the solvent evaporation method. The only difference between the two methods is that the drug solvent is miscible with the drug anti-solvent. Higher shear force prevents crystal growth and Ostwald ripening.

\section{Characterization of nanoemulsion [13-17] \\ Zeta potential}

Zeta potential is measured by an instrument known as Zeta PALS. It is used to measure the charge on the surface of the droplet in nanoemulsion. Emulsifiers not only act as a mechanical barrier but also through the formation of surface charges. Zeta potential can produce repulsive electrical forces among approaching oil droplets and this hinders coalescence. The more International Journal of Research in Pharmacy and Pharmaceutical Sciences 38 negative zeta potential, the greater the net charge of droplets and more stable the emulsion is. Zeta potential values lower than $-30 \mathrm{mV}$ generally indicate a high degree of physical stability. Malvern Zetasizer is based on dynamic light scattering and measures Zeta potential.

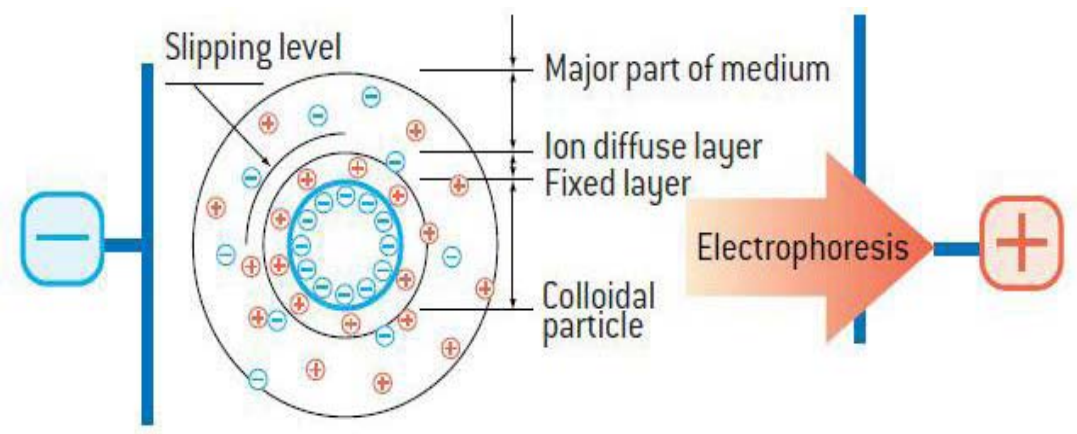

Fig. 2: Processes of zeta potential

\section{Polydispersity}

Polydispersity is the ratio of the standard deviation to mean droplet size, so it indicates the uniformity of droplet size within the formulation. The higher the polydispersity, the lower the uniformity of the droplet size in the formulation. Malvern Zetasizer is based on dynamic light scattering and measures polydispersity.

\section{Particle size analysis}

Generally, in case of nanoemulsion, dynamic light scattering (DLS) method is used for the measurement of particle size and their distribution.

\section{Percent of drug loading}

Pre-weighed nanoemulsion is extracted by dissolving in $25 \mathrm{ml}$ suitable solvent, an extract is then analyzed spectrophotometrically/HPLC. Against the standard solution of the drug. Drug content determines by reverse phase HPLC method using different columns of appropriate porosity.

\section{Transmission electron microscopy (TEM)}

Morphology and structure of the nanoemulsion can be studied using transmission electron microscopy (TEM).

\section{In vitro drug release}

The in vitro release studies of nanoemulsion containing drug can be investigated through semipermeable membrane used in a dissolution apparatus. A glass cylindrical tube $(2.5 \mathrm{~cm}$ in diameter and $6 \mathrm{~cm}$ in length) is attached instead of the basket and should tightly cover with the semi-permeable membrane. Drug-loaded nanoemulsion is placed in the cylindrical tube at the semi-permeable membrane surface. The cylindrical tube should dip in $100 \mathrm{ml}$ buffer maintaining the $\mathrm{pH}$ to allow the establishment of the sink conditions and to sustain permanent solubilization. The release study can be carried out for $24 \mathrm{~h}$. at $32 \mathrm{C}$. The stirring shaft should rotate at speed of 100 r. p. m. At predetermined time intervals $(1,2,4,6,8,12,20$, $24 \mathrm{~h}$.) aliquots of one milliliter of the release medium is withdrawn and diluted then filtered for analysis and replaced with an equal volume of the buffer solution to maintain a constant volume. The absorbance of the collected samples can be measured by UV spectrometer.

\section{Applications of nanoemulsions [18-24]}

\section{Parenteral delivery}

Nanoemulsion are advantages for intravenous administration, due to the strict requirement of this route of administration, particularly the 
necessity for the formulation droplet size lower than 1 micrometer. Parenteral (or Injectable) administration of nanoemulsion is employed for a variety of purposes, namely nutrition eg. Fats, Carbohydrates, Vitamins etc. Nanoemulsions of natural oils (soyabean, sesame and olive) with the nontoxic surfactant Pluronic F-68 via ultrasound for parenteral feeding. Lipidnanoemulsion has been widely explored for parenteral delivery of drugs. Nanoemulsion formulations have distinct advantages over macroemulsion systems when delivered parenterally because of the fine particle Nanoemulsion is cleared more slowly than the coarse particle emulsion and, therefore, have a longer residence time in the body. Both $\mathrm{O} / \mathrm{W}$ and $\mathrm{W} / 0$ Nanoemulsion can be used for parenteral delivery.

\section{Oral delivery}

Nanoemulsion formulations offer the several benefits over conventional oral formulation for oral administration including increased absorption, improved clinical potency and decreased drug toxicity. Therefore, Nanoemulsion has been reported to be ideal delivery of drugs such as steroids, hormones, diuretic and antibiotics. Pharmaceutical drugs of peptides and proteins are highly potent and specific in their physiological functions. Primaquine, when incorporated into oral lipid nanoemulsion, showed effective antimalarial activity against Plasmodium bergheii infection in mice at a $25 \%$ lower dose level as compared to the conventional oral dose. Lipidnanoemulsion of primaquine improved oral bioavailability by the liver with drug concentration higher at least by $45 \%$ as compared with the plain drug.

\section{Topical delivery}

Topical administration of drugs can have advantages over other methods for several reasons, one of which is the avoidance of hepatic first-pass metabolism of the drug and related toxicity effects. Another is the direct delivery and targetability of the drug to the affected area of the skin or eyes. The nanoemulsion can achieve a level of topical antimicrobial activity that has only been previously achieved by systemic antibiotics. The nanoemulsion has broadspectrum activity against bacteria (e. g. E. coli, S. aureus) fungi (e. g. Candida, Dermatophytes).

\section{Ocular delivery}

For the treatment of eye diseases, drugs are essentially delivered topically. O/W Nanoemulsions have been investigated for ocular administration, to dissolve poorly soluble drugs, to increase absorption and to attain prolong release profile.

\section{In cosmetic}

The aesthetic properties, i.e. low viscosity and transparent visual aspects of nanoemulsion with droplet sizes below $200 \mathrm{~nm}$, its high surface area allowing effective transport of the active ingredient to the skin make them especially attractive for their application in cosmetics. Nanoemulsions are acceptable in cosmetics because there is no inherent creaming, sedimentation, flocculation or coalescence that are observed with macroemulsion. The incorporation of potentially irritating surfactants can be avoided by using high energy equipment during manufacturing. Nanogel technology to create miniemulsion from oil-inwater concentrate suited to minimizing transepidermal water loss, enhanced skin protection and penetration of active ingredient. It would be useful for sun care products, moisturing and antiageing creams. It helps to give skin care formulations a good skin feels.

\section{Transdermal}

Indomethacin a potent NSAID, the anti-inflammatory effects of true optimized nanoemulsion formulation were compared with marketed gel in carrageenan induced paw edema in rats. The \%inhibition value was significant for developed Nanoemulsion, so great potential for transdermal application of indomethacin. Nanoemulsions for transdermal delivery of celecoxib. The formulation which consisted of $2 \%$ celecoxib $10 \%$ oil phase (Sefsol 218 and Triacetin) 50\% surfactant mixture (Tween 80 and Transcutol-P) and $40 \%$ water.

\section{In biotechnology}

Many enzymatic and biocatalytic reactions are conducted in pure organic or aqua-organic media. Biphasic media are also used for these types of reactions. The use of pure apolar media causes the denaturation of biocatalysts. The use of water-proof media is relatively advantageous. Enzymes in low water content display and have-

\author{
$>$ Increased solubility in non-polar reactants. \\ $>$ Possibility of shifting thermodynamic equilibria in favour of \\ condensations.
}

$>$ Improvement of thermal stability of the enzymes, enabling reactions to be carried out at higher temperatures.

\section{CONCLUSION}

Nanoemulsions propose several advantages for the delivery of drugs. They are applicable for almost all routes of delivery and therefore provide the promising effect for different fields like therapeutics, cosmetics and biotechnology. This new technology is developed to overcome the poor absorption of some pharmaceuticals and poor miscibility of these compounds with the lipid contents of cell membrane. The conventional approaches to bioavailability enhancement can hardly meet the challenges presented by the growing number of insoluble drugs. The effectiveness of nanoemulsion-based drug delivery in overcoming current bioavailability challenges are well documented in this literature. In this review, the modern strategies and considerations for successful nanoemulsion-based drug delivery have been presented with the hope that they can serve as the foundation for many more success in the field. The applications of nanoemulsion are limited by instability. Stability of formulation may be enhanced by controlling various factors such as type and concentration of surfactant and co-surfactant, type of oil phase, methods used, process variables and addition of additives used over the interphases of nanoemulsion formulation.

\section{AUTHORS CONTRIBUTIONS}

All the author have contributed equally

\section{CONFLICT OF INTERESTS}

Declare none

\section{REFERENCES}

1. Jain K, Kumar RS, Sood S, Gowthamarajan K. Enhanced oral bioavailability of atorvastatin via oil-in-water nanoemulsion using aqueous titration method. J Pharm Sci Res 2013;5:18-25.

2. Haritha, Basha SP, Rao KP, Chakravarthi V. A brief introduction to methods of preparation, applications and characterization of nanoemulsion drug delivery system. Indian J Res Pharm Biotechnol 2013;1:25-8.

3. Kumar SLH, Singh V. Nanoemulsification: a novel targeted drug delivery tool. J Drug Delivery Ther 2012;2:40-5.

4. Sole I, Pey CM, Maestro A, Gonzalez C, Porras M, Solans C, et al. Nanoemulsions prepared by phase inversion composition method: preparation variables and scale up. J Colloid Interface Sci 2010;344:417-23.

5. Ravi TPU, Padma T. Nanoemulsions for drug delivery through different routes. Res Biotechnol 2011;2:1-13.

6. Date AA, Nagarsenker S. Parenteral microemulsion: an overview. Int J Pharm 2008;355:19-30.

7. Sadurní N, Solans C, Azemar N, Garcia Celma MJ. Studies on the formation of $\mathrm{o} / \mathrm{w}$ nano-emulsions, by low energy methods, suitable for pharmaceutical applications. Eur J Pharm Appl 2005;26:438-45.

8. Sukanya G, Mantry S, Anjum S. Review on nanoemulsions. Int J Innovative Pharm Sci Res 2013;1:192-205.

9. Bhatt P, Madhav S. A detailed review on nanoemulsion drug delivery system. Int J Pharm Sci Res 2011;2:2482-9.

10. Jumaa M, Mueller BW. Formulation and stability of benzodiazepines in a new lipid emulsion formulation. Pharmazie 2002;57:740-3.

11. Shaji J, Joshi V. Self-microemulsifying drug delivery system (SMEDDS) for improving the bioavailability of hydrophobic drugs and its potential to give sustained release dosage forms. Indian J Pharm Educ 2005;39:130-5.

12. Carey MC, Small DM, Bliss CM. Lipid digestion and absorption. Ann Rev Physiol 1983;45:651-77. 
13. Narang AS, Delmarre D, Gao D. Stable drug encapsulation in micelles and microemulsions. Int J Pharm 2007;345:9-25.

14. Pouton CW, Porter CJH. Formation of lipid-based delivery systems for oral administration: materials, methods and strategies. Adv Drug Delivery Rev 2008;60:625-37.

15. Gursoy RN, Benita S. Self-emulsifying drug delivery systems (SEDDS) for improved oral delivery of lipophilic drugs. Biomed Pharmacother 2004;58:173-82.

16. Chime SA, Kenechukwu FC, Attama AA. Nanoemulsionsadvances in formulation, characterization and applications in drug delivery; 2014. p. 77-111.

17. Nirmala MJ, Shivashankar M, Mukherjee A, Chandrasekaran N Fluconazole: a simple nanoemulsion drug delivery system. Int J Pharm Pharm Sci 2013;5:716-7.

18. Thakur A, Walia MK, Kumar SLH. Nanoemulsion in the enhancement of bioavailability of poorly soluble drugs: a review. Int Res J 2013;4:15-25
19. Shah P, Bhalodia D, Shelat P. Nanoemulsion a pharmaceutical review. Systemic Rev Pharm 2010;1:24-32.

20. Singh BP, Kumar B, Jain SK, Shafaat K. Development and characterization of a nanoemulsion gel formulation for transdermal delivery of carvedilol. Int J Drug Dev Res 2012;4:151-61.

21. Heidi MM, Yun Seok R, Xiao W. Nanomedicine in pulmonary delivery. Int J Nanomed 2009;4:299-319.

22. Charles L, Attama AA. Current state of nanoemulsions in drug delivery. J Biomat Nanobiotech 2011;2:626-39.

23. Venkatesan N, Yoshimitsu J, Ito Y, Shibata N, Takada K. Liquid filled nanoparticles as a drug delivery tool for protein therapeutics. Biomaterials 2005;26:7154-63.

24. Kotta S, Khan AW, Pramod K, Ansari SH, Sharma RK, Ali J. Exploring oral nanoemulsions for bioavailability enhancement of poorly water-soluble drugs. Expert Opinion Drug Delivery 2012;9:585-98. 